\title{
Treponema Whipplei Endocarditis Masquerading as a Calcified Amorphous Aortic Valve Tumor
}

\author{
Elian Massoud ${ }^{1}$, Justin Watson ${ }^{2}$, and Amy Fiedler ${ }^{1}$ \\ ${ }^{1}$ University of Wisconsin \\ ${ }^{2}$ Oregon Health \& Science University School of Medicine
}

October 1, 2021

\begin{abstract}
Whipple's endocarditis is a rare culture-negative endocarditis caused by Tropheryma whipplei, an intracellular gram-positive organism. Here, we present a case of a 60-year-old male who presented with transient ischemic attack and was found to have an aortic valve mass. Following successful excision, histopathologic assessment of the lesion was consistent with calcified amorphous aortic tumor, a rare non-neoplastic hamartomatous mass of the heart. However, 16s rRNA and 18s rRNA sequencing detected Tropheryma whipplei, and the diagnosis of Whipple's endocarditis was made.
\end{abstract}

\section{Introduction}

Whipple's disease is a rare multi-system illness that classically presents with gastrointestinal symptoms, polyarthropathy, and central nervous system involvement in later stages [1]. Whipple Endocarditis is a rare cause of culture-negative subacute endocarditis. The first report of $T$. whipplei isolated from the aortic valve of a patient with blood-culture negative endocarditis was in 2000 [2]. Since then, 170 cases have been reported in the literature. It is not uncommon for Whipple endocarditis to present with arthralgia and heart failure without the classic features of Whipple's disease. The average age of presentation is 57 years, with male sex predominance. Valvular lesions most commonly involve the aortic valve, but multiple valves can also be infected [3].

On the other hand, calcified amorphous tumor (CAT) is a rare non-neoplastic hamartomatous mass of the heart. The majority of reported cases involve the mitral valve, with only a few reported cases arising from the aortic valve. Pathologically, these tumors are described as nodular calcium deposits with a background of fibrous tissue and chronic inflammatory cells [4]. Clinically, most patients are asymptomatic, but about $56 \%$ have obstructive symptoms, embolization, and/or syncope [5].

\section{Case Presentation}

A 60-year-old male with multiple medical including CLL, type 2 Diabetes Mellitus, hyperlipidemia, prostate cancer, and tobacco abuse, presented to the VA ED complaining of difficulty speaking and left arm weakness. No cardiac murmur was appreciated on physical exam and no focal neurologic deficits. Workup revealed a left parietal ischemic infarct. Further workup with Transthoracic Echocardiography revealed a trileaflet aortic valve with mild leaflet thickening and a calcified mobile mass on the aortic valve leaflets measuring 1.4 $\mathrm{cm}$. Evaluation with Transesophageal Echocardiogram confirmed the mass. Cardiac surgery was consulted for a suspected fibroelastoma. There was low suspicion for endocarditis with no evidence of a systemic infection. The mass was excised for histopathological evaluation consistent with a Calcified Amorphous Tumor (Fig. 1). However, 16s rRNA and 18s rRNA sequencing detected Tropheryma whipplei, and the diagnosis of Whipple's endocarditis was made. 


\section{Discussion}

The differential diagnosis for aortic valve mass includes papillary fibroelastoma, endocarditis, Lambl's excrescence. Our case highlights the challenge in diagnosing Whipple's endocarditis. Our patient presented with TIA from a cardiac embolic cause without arthralgia, the most common symptom of Whipple endocarditis, and no systemic signs of infection such as fever. However, the majority of cases described in the literature reported aortic valve lesions in the absence of fever [6]. Furthermore, it is crucial to recognize that $T$ whipplei endocarditis differs from classic Whipple disease, which tends to involve multiple organ systems. Our case also highlights the importance of molecular diagnostics in the diagnosis of that $T$ whipplei endocarditis. Pathologically, our patient's lesion resembled an Amorphous Cardiac Tumor, which rarely arises in the aortic valve. In fact, there have been four cases of CAT with isolated involvement of the aortic valve [7-10]. Several features made CAT an unlikely diagnosis in our patient. Even though pathogenesis of CAT is poorly understood, a systematic review revealed that $21 \%$ of CAT cases were found in patients with end-stage renal disease [11], which our patient did not have. Furthermore, there is a female predominance, and the majority of reported CAT cases involve the left ventricle the mitral valve, with only a few reported cases arising from the aortic valve [12]. A histopathologic diagnosis of $T$ whipplei endocarditis, however can be made with periodic acid-Schiff (PAS) staining. Although more commonly performed on duodenal biopsies in the diagnosis of classical whipple's disease, PAS-positive macrophages from an extracted valvular lesion has been reported $[6,13,14]$. For T whipplei endocarditis, molecular analysis with $16 \mathrm{~S}$ rRNA PCR following surgical resection of the valvular lesion is currently the preferred diagnostic approach. Unfortunately, $16 \mathrm{~S}$ rRNA amplification from blood specimens is not sensitive [15].

\section{Conclusion}

Tropheryma whipplei is a rare cause of culture-negative endocarditis. We report a case of $T$ whipplei endocarditis in the aortic valve masquerading as an amorphous cardiac tumor in a 60-year-old male treated by a complete resection of the mass. Further workup and medical knowledge, and risk stratification is needed for this relatively rare clinical entity.

\section{References}

1. "Whipple's Disease Information Page." National Institute of Neurological Disorders and Stroke, U.S. Department of Health and Human Services, www.ninds.nih.gov/Disorders/All-Disorders/Whipples-DiseaseInformation-Page.

2. Goldenberger D, Kunzli A, Vogt P, Zbinden R, Altwegg M. Molecular diagnosis of bacterial endocarditis by broad-range PCR amplification and direct sequencing. J Clin Microbiol. 1997;35:2733-9.

3. McGee, Michael, et al. "Tropheryma whipplei endocarditis: case presentation and review of the literature." Open forum infectious diseases. Vol. 6. No. 1. US: Oxford University Press, 2019.

4. Masuda, Shinya, et al. "Surgical removal of calcified amorphous tumor localized to mitral valve leaflet without mitral annular calcification." Surgical case reports 1.1 (2015): 1-5.

5. Raoult D, Birg M, La Scola B, Fournier P, Enea M, Lepidi H, et al. Cultivation of the bacillus of Whipple's disease. N Engl J Med. 2000;342:620-5. http://dx.doi.org/10.1056/NEJM 200003023420903

6. Fenollar, Florence, et al. "Tropheryma whipplei endocarditis." Emerging infectious diseases 19.11 (2013): 1721 .

7. Reynolds C, Tazelaar HD, Edwards WD. Calcified amorphous tumor of the heart (cardiac CAT). Hum Pathol. 1997;28:601-6.

8. Koyama Y, Tsujimoto S, Yamamoto M. Calcified amorphous tumor in the aortic valve identified by computed tomography. J Thorac Cardiovasc Surg. 2020 Mar;159(3):e161-e163. doi: 10.1016/j.jtcvs.2019.04.094. Epub 2019 May 16. PMID: 31202446. 
9. Fan J, Chen M, Bi S, Guo Y. Cardiac calcified amorphous tumor originating from the aortic valve: A rare case report. Anatol J Cardiol . 2018;19(3):E5-E6. doi:10.14744/AnatolJCardiol.2017.8244.

10. Yamamoto M, Nishimori H, Wariishi S, Fukutomi T, Kond N, Kihara K, Tashiro M, Tanioka K, Orihashi K. Cardiac calcified amorphous tumor stuck in the aortic valve that mimicked a chameleon's tongue: report of a case. Surg Today. 2014 Sep;44(9):1751-3. doi: 10.1007/s00595-013-0698-y. Epub 2013 Aug 28. PMID: 23982194.

11. De Hemptinne Q, de Cannière D, Vandenbossche JL, Unger P. Cardiac calcified amorphous tumor: a systematic review of the literature. Int J Cardiol Heart Vasc 2015;7:1-5.

12. Choi, Eunice K., Jae Y. Ro, and Alberto G. Ayala. "Calcified amorphous tumor of the heart: case report and review of the literature." Methodist DeBakey cardiovascular journal 10.1 (2014): 38.

13. McGee, Michael et al. "Tropheryma whipplei Endocarditis: Case Presentation and Review of the Literature." Open forum infectious diseases vol. 6,1 ofy330. 7 Dec. 2018, doi:10.1093/ofid/ofy330

14. Weisman, Alanna, et al. "Whipple's endocarditis: an enigmatic cause of culture-negative bacterial endocarditis." Canadian Journal of Infectious Diseases and Medical Microbiology 24.2 (2013): e29-e30.

15. Fournier, Pierre-Edouard, et al. "Comprehensive diagnostic strategy for blood culture-negative endocarditis: a prospective study of 819 new cases." Clinical Infectious Diseases 51.2 (2010): 131-140.

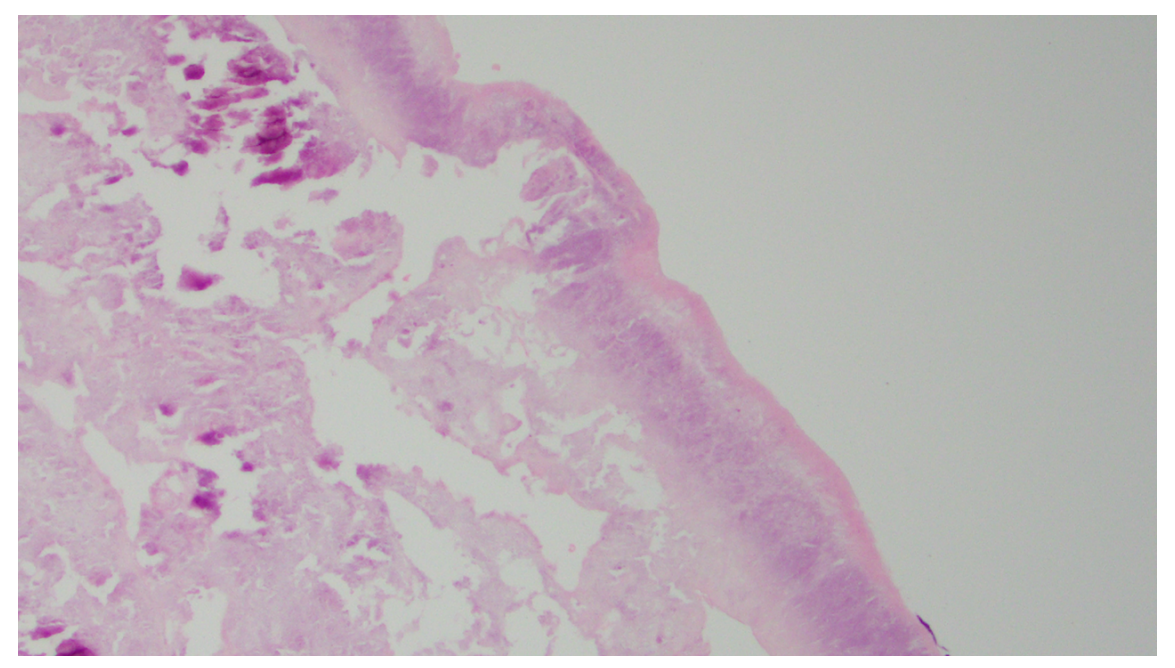

